\title{
Search for critical-point nuclei in terms of the sextic oscillator
}

\author{
G. Lévai* \\ Institute of Nuclear Research of the Hungarian Academy of Sciences (ATOMKI), Post Office Box 51, H-4001 Debrecen, Hungary \\ J. M. Arias ${ }^{\dagger}$ \\ Departamento de Física Atómica, Molecular y Nuclear, Facultad de Física, Universidad de Sevilla, Apartado 1065, E-41080 Sevilla, Spain
}

(Received 22 January 2010; published 7 April 2010)

\begin{abstract}
The spherical to deformed $\gamma$-unstable shape transition in nuclei is discussed in terms of the sextic oscillator as a $\gamma$-independent potential in the Bohr Hamiltonian. The wave functions, energy eigenvalues, and electric quadrupole and monopole transition rates are calculated in closed analytical form for the lowest-lying energy levels. It is shown that the locus of critical points for the spherical to deformed $\gamma$-unstable shape phase transition corresponds to a parabola in the parameter space of the model. The ratios of energy eigenvalues and electromagnetic transition probabilities are constant along this parabola. It is thus possible to associate parameter-free benchmark values to the ratios of relevant observables at the critical point of the transition that can be compared to experimental data. In addition, systematic studies of the shape evolution in isotope chains can be performed within the model. As an application, the model parameters are fitted to the energy spectra of the chains of even-even $\mathrm{Ru}, \mathrm{Pd}$, and $\mathrm{Cd}$ isotopes and the electric quadrupole transition probabilities are calculated. It is found that ${ }^{104} \mathrm{Ru},{ }^{102} \mathrm{Pd}$, and ${ }^{106,108} \mathrm{Cd}$ nuclei, which are usually considered to be good candidates for the $E(5)$ symmetry, lie rather close to the critical parabola that separates the spherical and deformed $\gamma$-unstable domains. The isotope ${ }^{116} \mathrm{Cd}$ is proposed as a new candidate for a similar critical-point nucleus.
\end{abstract}

DOI: 10.1103/PhysRevC.81.044304

PACS number(s): 21.60.Ev, 21.10.Re, 27.60.+j, 03.65.Ge

\section{INTRODUCTION}

The collective motion in nuclei is governed by the competition of the short-range pairing interaction, which favors spherical shapes, and the long-range quadrupole-quadrupole interaction, which induces deformation. The balance between these forces produces for a given nucleus a particular shape. Because this balance depends on the nucleon number, a major issue in nuclear structure physics is to understand the evolution of the nuclear shapes as the nucleon number is varied. From a phenomenological point of view, this can be done either within the collective model [1], solving the corresponding Bohr Hamiltonian, or within the interacting boson model (IBM) [2] that considers the nucleus as a boson system in which only valence nucleons coupled by pairs to angular momenta $L=0$ and $L=2$ are active. Both approaches can describe the main different shapes observed in nuclei: spherical, axially deformed, and deformed $\gamma$-unstable shapes. In the IBM case, these shapes correspond to the three well-known dynamical symmetries of the model: the $\mathrm{U}(5), \mathrm{SU}(3)$, and $\mathrm{O}(6)$ symmetries, respectively [2]. There have been many studies establishing the phase diagram of the IBM including the dynamical symmetries and the transitions between them; see, for instance, recent review articles, Refs. [3-7]. An alternative, as mentioned previously, and rather effective method for accomplishing the same study is the application of the Bohr Hamiltonian, which uses a potential picture to describe quadrupole collective excitations in terms of the intrinsic shape variables $\beta, \gamma$. The absolute minimum of the $V(\beta, \gamma)$ potential defines the equilibrium

\footnotetext{
*levai@ atomki.hu

†ariasc@us.es
}

shape, while the potential itself depends on various parameters characterizing the given nucleus, for example, the proton and neutron numbers, etc. Changing these parameters gradually (e.g., by proceeding along an isotope chain), the potential shape and its minimum change and may give rise to a transition from one equilibrium shape (and symmetry) to another one. This procedure can be interpreted as a shape phase transition of some order that goes through a critical point. In this article we are interested in modeling such a critical point for the second-order phase transition from spherical to deformed $\gamma$-unstable shapes. The study is done in the framework of the collective model in which the differential Bohr equation has to be solved with a potential that is independent on the $\gamma$ variable. In this case, the Bohr Hamiltonian reduces to a Schrödinger-like radial equation. Several studies have tried to characterize the critical point in this transition. The reference one was proposed by Iachello, who introduced the concept of the $E(5)$ critical point symmetry [8]. At the $E(5)$ critical point, the potential proposed is $\gamma$-independent plus an infinite square well in the $\beta$ variable [8], mimicking the situation in which a second-order phase transition occurs between the spherical and the $\gamma$-unstable phases. This problem can be solved exactly in terms of Bessel functions, so parameter-free analytical results are available for the key spectroscopic properties (energy spectrum, electric quadrupole transition rates). Comparison of these quantities with the spectroscopic data of actual nuclei revealed that several nuclei are close to the $E(5)$ critical-point symmetry. The first example was the ${ }^{134} \mathrm{Ba}$ nucleus [9]. Soon after, the ${ }^{104} \mathrm{Ru}[10]$ and ${ }^{102} \mathrm{Pd}[11]$ nuclei were also discussed in terms of this scheme. Later, in a systematic search for $E(5)$ candidates, the ${ }^{106} \mathrm{Cd},{ }^{108} \mathrm{Cd},{ }^{124} \mathrm{Te}$, and ${ }^{128} \mathrm{Xe}$ nuclei were also proposed [12]. More recently, the ${ }^{124} \mathrm{Xe}$ isotope has been mentioned as a promising candidate [13]. 
Besides $E(5)$, a new critical point symmetry, called X(5), has also been introduced [14] to give account of the first-order phase transition between the spherical and axially deformed shape phases. In this case, the $\beta$ degree of freedom is also modeled with an infinite square well but now the $\gamma$ shape variable has to be incorporated into the formalism through certain approximation schemes [14].

For the case of $\gamma$-independent potentials, in addition to the infinite square well for the $\beta$ variable, there are few potentials with which the Bohr Hamiltonian (or the one-dimensional equation derived from it) can be solved exactly. These cases are typically solvable radial problems in three-dimensions with a modified centrifugal term that accounts for changing the spatial dimension from three to five. To obtain a complete picture, potentials with a centrifugal-like term have to be considered. The list of exactly solvable problems of this type is finite (see, e.g., Ref. [15]). Besides the infinite square well, only the Kratzer and Davidson potentials (the generalizations of the Coulomb and harmonic oscillator potentials) can be solved for arbitrary quantum numbers. Recently, we introduced a rather flexible potential [16], which is quasi-exactly solvable. This means that closed analytical solutions are available only for a finite subset of states for certain potential parameters. However, this set of states contains the most important lowlying levels necessary to characterize the collective excitations of the nucleus within the scheme of the Bohr Hamiltonian. This potential is the sextic oscillator and can describe situations in which a nucleus has a spherical minimum (at $\beta=0$ ), a deformed minimum (at $\beta>0$ ), or both. This feature is certainly advantageous when one wishes to describe transitions between different shape phases. In its first application, the sextic oscillator was just shown to be a $\gamma$-independent potential appropriate for describing the transition between the spherical and the $\gamma$-unstable shape phases. Here we show that this transition corresponds to crossing a parabola in the parameter space of the model and that the ratios of energy eigenvalues and electromagnetic transition probabilities are constant along this parabola. This produces parameter-free benchmark values for the ratios of relevant observables, which can be compared with experimental data and provides another independent way of looking for critical nuclei in the spherical to deformed $\gamma$-unstable shape phase transition. In addition, we present a systematic study of the evolution from spherical to deformed $\gamma$-unstable shapes in the $\mathrm{Ru}, \mathrm{Pd}$, and $\mathrm{Cd}$ isotope chains by using the sextic oscillator as a $\gamma$-independent potential in the Bohr equation. The wave functions, energy eigenvalues, and electric quadrupole and monopole transition rates are calculated in closed analytical form for the lowest-lying energy levels. The sextic oscillator has already been applied to ${ }^{134} \mathrm{Ba}$, not in a full scale fit, but rather as an illustration of the model [16]. Preliminary results for the $\mathrm{Ru}$ isotopes have already been presented [17,18].

The article is structured as follows. In Sec. II, the quasiexactly solvable sextic potential is briefly discussed and closed analytical forms are obtained for wave functions, energies, and $E 2$ and $E 0$ electromagnetic transitions involving the relevant low-lying states in nuclei. In Sec. III, the benchmark calculation for relevant observables at the critical point, modeled with the sextic oscillator potential, in the spherical to deformed $\gamma$-unstable shape phase transition is given. The application of the sextic potential to the $\mathrm{Ru}, \mathrm{Pd}$, and Cd isotope chains is presented and discussed in Secs. IV and V. Finally, Sec. VI is devoted to summarizing the main results. At the end, there is a mathematical appendix with details for the analytical evaluation of the relevant integrals appearing in Sec. II.

\section{THE SEXTIC OSCILLATOR}

The Bohr Hamiltonian is written as [1]

$$
\begin{aligned}
H= & -\frac{\hbar^{2}}{2 B}\left[\frac{1}{\beta^{4}} \frac{\partial}{\partial \beta} \beta^{4} \frac{\partial}{\partial \beta}+\frac{1}{\beta^{2} \sin 3 \gamma} \frac{\partial}{\partial \gamma} \sin 3 \gamma \frac{\partial}{\partial \gamma}\right. \\
& \left.-\frac{1}{4 \beta^{2}} \sum_{k} \frac{Q_{k}^{2}}{\sin ^{2}\left(\gamma-\frac{2}{3} \pi k\right)}\right]+V(\beta, \gamma) .
\end{aligned}
$$

If the potential is assumed to be independent on the $\gamma$ variable, that is, $V(\beta, \gamma)=U(\beta)$, then the $\beta$-dependent part can be separated by the substitution $\Psi\left(\beta, \gamma, \theta_{i}\right)=\beta^{-2} \phi(\beta) \Phi\left(\gamma, \theta_{i}\right)$, leading to a form similar to the usual radial Schrödinger equation

$$
-\frac{d^{2} \phi}{d \beta^{2}}+\left[\frac{(\tau+1)(\tau+2)}{\beta^{2}}+u(\beta)\right] \phi=\epsilon \phi,
$$

where $\epsilon=\frac{2 B}{\hbar^{2}} E$ and $u(\beta)=\frac{2 B}{\hbar^{2}} U(\beta)$. In Eq. (2), $\tau$ originates from the angular equation and essentially plays the role of the angular momentum in five spatial dimensions. The differential equation for the $\gamma$ and Euler angles part was already solved in Ref. [19] for a limited set of angular momenta and more recently in general form in Ref. [20].

In Ref. [16] the $u(\beta)$ potential was chosen as the sextic oscillator

$$
u(\beta)=\left(b^{2}-4 a c^{\pi}\right) \beta^{2}+2 a b \beta^{4}+a^{2} \beta^{6}+u_{0}^{\pi},
$$

which is a quasi-exactly solvable potential [21], meaning that only a subset of its lowest-energy solutions can be obtained exactly for certain combinations of the potential parameters.

$\gamma$-independent potentials with the structure of Eq. (3) can be obtained from the IBM using intrinsic coherent states including three-body terms. The $\beta^{2}$ and $\beta^{4}$ terms are obtained from the usual one- and two-body terms $\widehat{n}_{d}, \widehat{P}^{\dagger} \widehat{P}$, and $\widehat{Q} \cdot \widehat{Q}$, with $\chi=0$ (see Ref. [2] for notation), while the $\beta^{6}$ term is obtained, for instance, with the three-body term $\left(d^{\dagger} d^{\dagger} d^{\dagger}\right)^{(2)} \cdot(\tilde{d} \tilde{d} \tilde{d})^{(2)}$.

Before discussing in more detail the importance of quasiexactly solvability and that of the parameters, it is worthwhile to examine the possible minima and maxima of the potential in Eq. (3). The extrema of the potential depend on the sign of $b$ and $b^{2}-4 a c^{\pi}$, that is, on the coefficients of the $\beta^{2}$ and $\beta^{4}$ terms in Eq. (3). If $b^{2}>4 a c^{\pi}$ and $b>0$ hold, then the potential has a minimum at $\beta=0$ and it increases with $\beta$. When $b^{2}<4 a c^{\pi}$ (irrespective of the sign of $b$ ), the minimum shifts to a finite value of $\beta$, while for $b^{2}>4 a c^{\pi}$ and $b<0$ [i.e., $\left.b<-2\left(a c^{\pi}\right)^{1 / 2}\right]$, there are two minima (one at $b=0$ and one at $\beta>0$ ) separated by a local maximum. The $b^{2}=4 a c^{\pi}$ parabola in Fig. 1 thus separates the $a(b)$ plane into three domains: to the right of it the minimum is spherical $\left(\beta_{\min }=0\right)$, above the parabola the minimum is deformed $\left(\beta_{\min }>0\right)$, and 


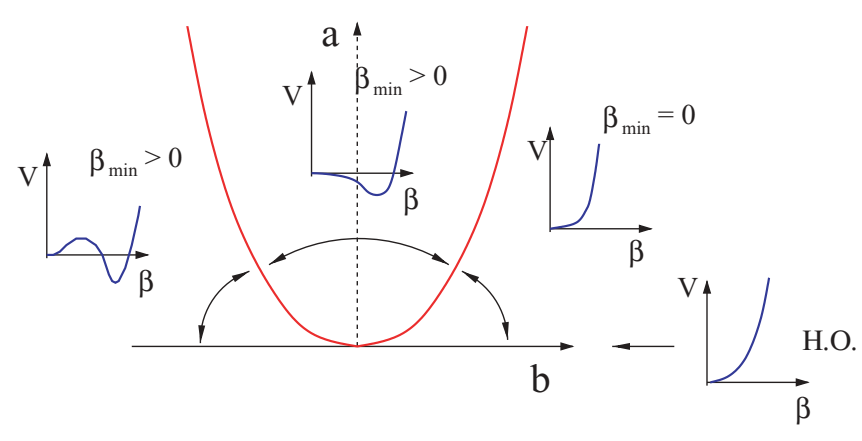

FIG. 1. (Color online) Domains of the $(a, b)$ parameter space corresponding to specific potential shapes. The critical parabola is $a=b^{2} / 11$. The positive $b$ axis corresponds to the harmonic oscillator.

to the left there are two minima such that $\left(\beta_{\min 2}>\beta_{\min 1}=0\right)$. Crossing the right leg of the parabola thus corresponds to the transition from the spherical to the deformed phase, where the potential is rather flat, so it is expected to be close to the $E(5)$ critical point. The domain to the left of the parabola corresponds to potential shapes typical for the X(5) symmetry so the sextic oscillator may also be appropriate to describe the $\beta$-dependent part of the $V(\beta, \gamma)$ potential of such systems. The exact locations of the extrema are determined by the relation

$$
\left(\beta_{0}^{\pi}\right)^{2}=\frac{1}{3 a}\left[-2 b \pm\left(b^{2}+12 a c^{\pi}\right)^{1 / 2}\right] .
$$

Whenever the right-hand side of Eq. (4) is positive, the "+" sign determines the nonzero minimum, while the "-" sign sets the maximum for $b<0$.

The $u(\beta)$ potential in Eq. (3) depends on three parameters, $a, b$, and $c^{\pi}$, but $c^{\pi}$ is related to the $\tau$ quantum number via $2 c^{\pi}=\left(\tau+2 M+\frac{7}{2}\right)$ in order to account for the "centrifugal" term. Here $M$ is a non-negative integer number that sets the number $M+1$ of solutions that can be obtained exactly. In typical applications of the sextic oscillator in the Bohr Hamiltonian, it is sufficient to consider $M=0$ and $M=1$ : with these choices the most characteristic levels are included in the description. The potential is slightly different for even and odd values of $\tau$ (or the $\tau+2 M$ combination): in the former case, $c^{+}=11 / 4$, while in the latter one, $c^{-}=13 / 4$, corresponding to $\tau+2 M=2$ and 3 , respectively. This slight difference, which resembles the parity dependence of radial potentials in three spatial dimensions manifests itself near $\beta=0$ in the coefficient of the $\beta^{2}$ term. In Ref. [16] this slight ambiguity was handled by selecting the constant terms $u_{0}^{+}$ and $u_{0}^{-}$such that the minima of the two potentials are set at the same energy. This requirement can be fulfilled by setting $u_{0}^{+}=0$ and

$$
u_{0}^{-}= \begin{cases}\left(b^{2}-11 a\right)\left(\beta_{0}^{+}\right)^{2}-\left(b^{2}-13 a\right)\left(\beta_{0}^{-}\right)^{2}+2 a b\left[\left(\beta_{0}^{+}\right)^{4}-\left(\beta_{0}^{-}\right)^{4}\right]+a^{2}\left[\left(\beta_{0}^{+}\right)^{6}-\left(\beta_{0}^{-}\right)^{6}\right] & \text { if } b^{2}<11 a, \\ -\left(b^{2}-13 a\right)\left(\beta_{0}^{-}\right)^{2}-2 a b\left(\beta_{0}^{-}\right)^{4}-a^{2}\left(\beta_{0}^{-}\right)^{6} & \text { if } 11 a<b^{2}<13 a, \\ 0 & \text { if } \quad b^{2}>13 a,\end{cases}
$$

where $\beta_{0}^{ \pm}$is the location of the nonzero minimum of $u^{ \pm}(\beta)$ discussed previously.

The solutions of Eq. (2) with the potential (3) are written as

$$
\phi_{\alpha}(\beta)=N_{\alpha} \beta^{p_{\alpha}}\left(1+d_{\alpha} \beta^{2}\right) \exp \left(-\frac{a}{4} \beta^{4}-\frac{b}{2} \beta^{2}\right),
$$

where $\alpha$ stands for the quantum numbers $\xi, \tau$ (the notation follows Refs. [14,16]). Table I contains the coefficients $p_{\alpha}$ and $d_{\alpha}$ for each wave function, together with the corresponding energy eigenvalue. The $d_{\alpha}$ coefficients there contain the following expressions:

$$
\lambda_{ \pm}=2 b \pm 2\left(b^{2}+10 a\right)^{1 / 2} ; \quad \tilde{\lambda}_{ \pm}=2 b \pm 2\left(b^{2}+14 a\right)^{1 / 2} .
$$

In Fig. 1 of Ref. [16] a schematic spectrum of the low-lying levels obtained with the sextic oscillator with indication of the relevant quantum numbers is displayed.

Note that normalizability requires $a \geqslant 0$ and that $a=0$ corresponds to the harmonic oscillator limit. Note also that the $a$ and $b$ parameters can be fitted exactly to two energy eigenvalues as

$$
a=E_{1,2}^{*}\left[E_{2,0}^{*}-E_{1,2}^{*}\right] / 40, \quad b=\left[2 E_{1,2}^{*}-E_{2,0}^{*}\right] / 4 .
$$

In practice, this means that knowing the energies of the $0^{+}$ state with $\xi=2$ and that of the $2_{2}^{+}$or $4_{1}^{+}$state (with $\xi=1$ and $\tau=2$ ) immediately provides us with a first approximation of the model parameters $(a, b)$.

The $N_{\alpha} \equiv N_{\xi \tau}$ normalization constants can be evaluated analytically using Eq. (A7) in the Appendix for the special case $W_{\alpha \alpha}^{0}=1$, and the result is

$$
\begin{aligned}
N_{\alpha}= & (2 a)^{\left(2 p_{\alpha}+1\right) / 8}\left[\frac{2}{\Gamma\left(p_{\alpha}+\frac{1}{2}\right)}\right]^{1 / 2}\left\{U\left(\frac{1}{4}\left(2 p_{\alpha}+1\right), \frac{1}{2} ; \frac{b^{2}}{2 a}\right)\right. \\
& +\left(p_{\alpha}+\frac{1}{2}\right) 2 \frac{d_{\alpha}}{(2 a)^{1 / 2}} U\left(\frac{1}{4}\left(2 p_{\alpha}+3\right), \frac{1}{2} ; \frac{b^{2}}{2 a}\right) \\
& +\left(p_{\alpha}+\frac{1}{2}\right)\left(p_{\alpha}+\frac{3}{2}\right)\left[\frac{d_{\alpha}}{(2 a)^{1 / 2}}\right]^{2} \\
& \left.\times U\left(\frac{1}{4}\left(2 p_{\alpha}+5\right), \frac{1}{2} ; \frac{b^{2}}{2 a}\right)\right\}^{-1 / 2}
\end{aligned}
$$


TABLE I. Explicit form of the lowest few energy eigenvalues and the coefficients appearing in the wave functions. Here $u_{0}^{-}$is defined in Eq. (5).

\begin{tabular}{cccccrc}
\hline \hline$\xi$ & $\tau$ & $M$ & $E_{\xi, \tau}$ & $E_{\xi, \tau}^{*}=E_{\xi, \tau}-E_{1,0}$ & $p_{\xi, \tau}=\tau+2$ \\
\hline 1 & 0 & 1 & $5 b+\lambda_{-}$ & 0 & 2 \\
1 & 1 & 1 & $7 b+\tilde{\lambda}_{-}+u_{0}^{-}$ & $2 b+\tilde{\lambda}_{-}-\lambda_{-}+u_{0}^{-}$ & 3 & $-\lambda_{-} / 10$ \\
1 & 2 & 0 & $9 b$ & $4 b-\lambda_{-}$ & 4 & $-\tilde{\lambda}_{-} / 14$ \\
1 & 3 & 0 & $11 b+u_{0}^{-}$ & $6 b-\lambda_{-}+u_{0}^{-}$ & 0 \\
2 & 0 & 1 & $5 b+\lambda_{+}$ & $\lambda_{+}-\lambda_{-}$ & 0 \\
2 & 1 & 1 & $7 b+\tilde{\lambda}_{+}+u_{0}^{-}$ & $2 b+\tilde{\lambda}_{+}-\lambda_{-}+u_{0}^{-}$ & 2 & 3 \\
\hline \hline
\end{tabular}

where $U$ are Kummer's functions, which are closely related to the well-known confluent hypergeometric functions [22]. Equation (A7) can also be used to evaluate the general overlap integrals of any pair of radial wave functions just taking $\delta=0$. To guarantee the orthogonality of all wave functions, one has to check just the cases when the $\tau$ quantum numbers of the two overlapping wave functions are equal, while their $\xi$ quantum numbers are different (in all other cases, the $\tau$-dependent component of the wave function will guarantee the orthogonality of the total wave functions). Making use of Eqs. (A4) and (A5), straightforward algebra proves that $W_{10,20}^{0}=0$ and $W_{11,21}^{0}=0$, that is, the orthogonality of the different radial wave functions belonging to $\tau=0$ and 1 .

The radial integrals necessary to evaluate electromagnetic transition rates can also be calculated analytically using Eq. (A7) with the appropriate $\delta$ value. In particular, using the first-order electric quadrupole transition operator $[8,23]$

$$
T^{(E 2)}=t \beta\left\{D_{\mu, 0}^{(2)} \cos \gamma+2^{-1 / 2}\left[D_{\mu, 2}^{(2)}+D_{\mu,-2}^{(2)}\right] \sin \gamma\right\},
$$

the relevant radial matrix elements are

$$
\begin{aligned}
M(E 2 ; \alpha \rightarrow \gamma) & \\
= & W_{\alpha, \gamma}^{1}=N_{\alpha} N_{\gamma} \frac{1}{2} \Gamma\left(\frac{p_{\alpha}+p_{\gamma}+2}{2}\right)(2 a)^{-\left(p_{\alpha}+p_{\gamma}+2\right) / 4} \\
& \times\left\{U\left(\frac{1}{4}\left(p_{\alpha}+p_{\gamma}+2\right), \frac{1}{2} ; \frac{b^{2}}{2 a}\right)+\frac{p_{\alpha}+p_{\gamma}+2}{2}\right. \\
& \times \frac{d_{\alpha}+d_{\gamma}}{(2 a)^{1 / 2}} U\left(\frac{1}{4}\left(p_{\alpha}+p_{\gamma}+4\right), \frac{1}{2} ; \frac{b^{2}}{2 a}\right) \\
& +\frac{p_{\alpha}+p_{\gamma}+2}{2} \frac{p_{\alpha}+p_{\gamma}+4}{2} \frac{d_{\alpha} d_{\gamma}}{2 a} \\
& \left.\times U\left(\frac{1}{4}\left(p_{\alpha}+p_{\gamma}+6\right), \frac{1}{2} ; \frac{b^{2}}{2 a}\right)\right\}
\end{aligned}
$$

It is enough to calculate the integrals with $\Delta \tau= \pm 1$, because the remaining matrix elements will be zero due to the $\tau$ dependent components of the total wave functions. It should be noted that to get the corresponding transition probabilities, the preceding radial integrals should be evaluated as well as those in the $\gamma$ and Euler angle parts.

For the electric monopole transitions, the $\delta=2$ case of Eq. (A7) has to be considered because the monopole transition operator is proportional to $\beta^{2}$,

$$
T^{(E 0)}=q \beta^{2} .
$$

The relevant radial matrix elements are

$$
\begin{aligned}
M(E & ; \alpha \rightarrow \gamma) \\
= & W_{\alpha, \gamma}^{2}=N_{\alpha} N_{\gamma} \frac{1}{2} \Gamma\left(\frac{p_{\alpha}+p_{\gamma}+3}{2}\right)(2 a)^{-\left(p_{\alpha}+p_{\gamma}+3\right) / 4} \\
& \times\left\{U\left(\frac{1}{4}\left(p_{\alpha}+p_{\gamma}+3\right), \frac{1}{2} ; \frac{b^{2}}{2 a}\right)+\frac{p_{\alpha}+p_{\gamma}+3}{2}\right. \\
& \times \frac{d_{\alpha}+d_{\gamma}}{(2 a)^{1 / 2}} U\left(\frac{1}{4}\left(p_{\alpha}+p_{\gamma}+5\right), \frac{1}{2} ; \frac{b^{2}}{2 a}\right) \\
& +\frac{p_{\alpha}+p_{\gamma}+3}{2} \frac{p_{\alpha}+p_{\gamma}+5}{2} \frac{d_{\alpha} d_{\gamma}}{2 a} \\
& \left.\times U\left(\frac{1}{4}\left(p_{\alpha}+p_{\gamma}+7\right), \frac{1}{2} ; \frac{b^{2}}{2 a}\right)\right\}
\end{aligned}
$$

Here again the radial integrals have to be combined with those in the $\tau$ and Euler angle part, and the appropriate selection rules ( $\Delta \tau=0, \Delta J=0$ ) also have to be taken into consideration.

The $W_{\alpha \gamma}^{2}$ quantities can also be used to calculate the matrix elements of the second-order term of the electric quadrupole operator [24]. In that case, a wider variety of radial integrals have to be calculated, because of the different selection rules in the $\tau$ and angular momentum quantum numbers. The main effect of the inclusion of a quadratic term in the $E 2$ operator is that transitions with $\Delta \tau= \pm 2$ are not forbidden, although, for reasonable values of the parameter giving the relative weight of the quadratic to the linear terms in the $E 2$ operator, they are much smaller than the $\Delta \tau= \pm 1$ transitions. In this article, only the linear term in the $E 2$ operator is considered, although the inclusion of the quadratic one is straightforward using Eq. (A7) with $\delta=2$.

\section{BENCHMARK QUANTITIES AT THE CRITICAL POINT OF THE SPHERICAL TO DEFORMED $\gamma$-UNSTABLE SHAPE PHASE TRANSITION}

As mentioned previously, the branch with $b>0$ of the $b^{2}=11 a$ parabola plays a special role in the parameter space, as it separates the domains in which the system has spherical potential minimum (at $\beta_{0}=0$ ) from that in which the potential minimum belongs to finite deformation $\left(\beta_{0}>0\right)$. Because the quadratic potential term vanishes in this case, the potential is rather flat along the parabola. A closer look at the energy eigenvalues and the matrix elements [Eq. (A7)] reveals that these quantities depend on the $a$ and $b$ parameters in a 
TABLE II. Ratios of some energy eigenvalues and electric quadrupole transition strengths from the sextic oscillator with parameters along the critical parabola, the infinite square well [8], and the pure $\beta^{4}$ and $\beta^{6}$ potentials [25].

\begin{tabular}{lcccccc}
\hline \hline & $E\left(4_{1,2}^{+}\right)$ & $\frac{E\left(0_{2,0}^{+}\right)}{E\left(2_{1,1}^{+}\right)}$ & $\frac{E\left(6_{1,3}^{+}\right)}{E\left(2_{1,1}^{+}\right)}$ & $\frac{B\left(E 2 ; 4_{1,2}^{+} \rightarrow 2_{1,1}^{+}\right)}{E\left(2_{1,1}^{+}\right)}$ & $\frac{B\left(E 2 ; 0_{2,0}^{+} \rightarrow 2_{1,1}^{+}\right)}{B\left(E 2 ; 2_{1,1}^{+} \rightarrow 0_{1,0}^{+}\right)}$ & $\frac{B\left(E 2 ; 0_{1,3}^{+} \rightarrow 2_{1,2}^{+}\right)}{B\left(E 2 ; 2_{1,1}^{+} \rightarrow 0_{1,0}^{+}\right)}$ \\
\hline $\mathrm{U}(5)$ & 2.00 & 2.00 & 3.00 & 2.00 & 2.00 \\
$\beta^{4}$ & 2.09 & 2.39 & 3.27 & 1.83 & 1.42 & 1.19 \\
$\beta^{6}$ & 2.14 & 2.62 & 3.39 & 1.77 & 0.87 \\
$E(5)$ & 2.20 & 3.03 & 3.59 & 1.68 & 1.59 \\
Sextic osc. & 2.66 & 3.08 & 3.80 & 1.87 & 2.56 \\
\hline \hline
\end{tabular}

particular form that makes it possible to identify parameterfree benchmark quantities for the critical point at the spherical to deformed $\gamma$-unstable phase transition within the framework of the sextic oscillator.

For this, first we observe that in the argument of the confluent hypergeometric functions in Eq. (A7) always the $b^{2} /(2 a)$ quantity occurs, which is constant along any parabola, including also the critical one at $b^{2}=11 a$. Then we also observe that the remaining expressions within the parentheses in Eq. (A7) also depend only on the $b^{2} /(2 a)$ combination. To see this, one has to compare the $d_{\alpha}(2 a)^{-1 / 2}$ quantities, where the $d_{\alpha}$ coefficients are defined in Table I in terms of the expressions in Eq. (7). The numerical factor outside the parenthesis in Eq. (A7), however, scales with $2 a$ in a characteristic way. Note that this is also true for the $N_{\alpha}$ normalization constants [Eq. (9)]. Collecting all the $a$ dependent terms it turns out that the scaling property of the general integral [Eq. (A7)] is $W_{\alpha, \gamma}^{\delta} \sim(2 a)^{-\delta / 4} F\left[b^{2} /(2 a)\right]$. In particular, the $B(E 2)$ and $B(E 0)$ transition ratios scale with $a$ as $a^{-1 / 2}$ and $a^{-1}$, respectively.

From this it follows that any ratio of $B(E 2)$ [and $B(E 0)$ ] values will depend exclusively on $b^{2} /(2 a)$; that is, they will be constant along the critical parabola. This also holds for any energy ratio, as can be seen from Table I and Eqs. (5) and (7). This means that the critical phase transition can be associated to parameter-free ratios, exactly as it was done in the case of the five-dimensional infinite well that defined the $E(5)$ critical point symmetry [8]. Figure 2 displays these benchmark numbers.

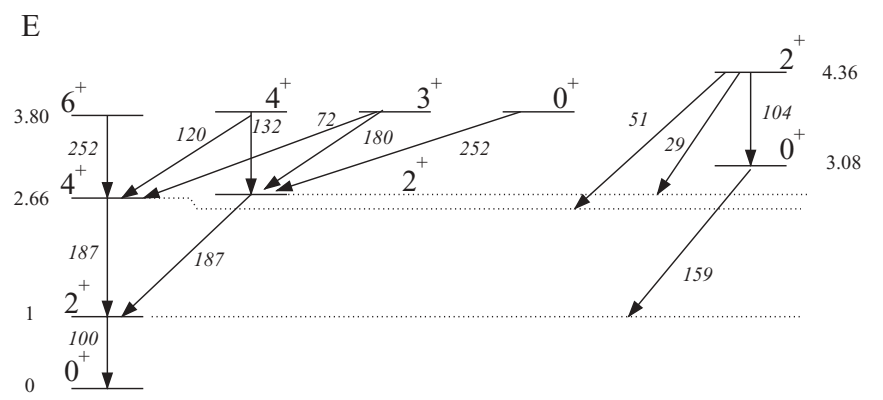

FIG. 2. Benchmark numbers [energies normalized to the excitation energy of the first $2^{+}$and $B(E 2)$ values normalized to $B\left(E 2 ; 2_{1}^{+} \rightarrow 0_{1}^{+}\right)=100$ ] characterizing the transition from the spherical to the deformed domain within the sextic oscillator model. These quantities are fixed for any point of the critical parabola separating the two domains.
Table II displays some benchmark values (the ratios of energy eigenvalues and electric quadrupole transition rates) extracted from the sextic oscillator (along the critical parabola) and various other models, for example, the five-dimensional harmonic oscillator, the pure quartic and pure sextic anharmonic oscillators, and the five-dimensional infinite well.

As has been mentioned previously, the sextic oscillator along the critical parabola contains only quartic and sextic terms. It is seen that the $B(E 2)$ ratios of the model are close to those of the $\beta^{4}$ model, which indicates that the effect of the additional sextic potential term may be less significant in affecting the wave functions than in changing the energy spectrum.

The energy ratios seem to be somewhat higher than those in the other models, the explanation of which arises naturally from the structure of Eq. (3). As has been mentioned before, the potential is slightly different for $\tau$-even and $\tau$-odd states owing to the difference in $c^{+}=11 / 4$ and $c^{-}=13 / 4$. This means that the quadratic component of the latter potential contains an extra $-2 a \beta^{2}$ term, which makes it somewhat deeper, pushing the $\tau$-even levels deeper [although this is partly compensated by the choice of $u_{0}^{-}$in Eq. (5)]. Because the reference level, the first $2^{+}$state, also belongs to odd $\tau$ [with quantum numbers $(\xi, \tau)=(1,1)]$, the energy ratios move somewhat upward.

This duality of the potential also manifests itself in the fact that there is a separate critical parabola for the $\tau$-odd states $a=$ $b^{2} / 13$, which lies somewhat below the critical parabola for the $\tau$-even states, $a=b^{2} / 11$. It is, however, reasonable to consider the $a=b^{2} / 11$ parabola as the critical one, because the $\tau$-even part of the spectrum contains the ground state and the excited $0^{+}$state with $(\xi, \tau)=(2,0)$, which plays an essential role in the quadrupole collective excitations. On the other hand, the $\tau$ odd part of the spectrum contains the $(\xi, \tau)=(1,3)$ multiplet that includes (in principle) degenerate states with $J^{\pi}=0^{+}$, $3^{+}, 4^{+}$, and $6^{+}$, the experimental correspondents of which usually do not follow this pattern. Associating a potential to these states [and also to the higher lying $(\xi, \tau)=(2,1) 2^{+}$ state] can thus be done in a less well-established way. The expected degeneracy of the $\tau$-even states $(\xi, \tau)=(1,2)$ with $J^{\pi}=2^{+}$and $4^{+}$is usually much better realized in experiment.

\section{APPLICATION TO Ru, Pd, AND Cd NUCLEI}

The region around the $Z=50$ proton closed shell seems to contain the best candidates for critical nuclei at the spherical to deformed $\gamma$-unstable shape phase transition. Here we consider isotope chains below this shell closure and analyze 
nuclei in the $\mathrm{Ru}, \mathrm{Pd}$, and $\mathrm{Cd}$ isotope chains to identify which are the critical nuclei in these chains from the sextic oscillator potential formalism presented here. This analysis is an extension of that in Ref. [26] in several respects: In addition to the wider range of isotopes considered, a more complete compilation of the experimental data is taken; furthermore, the electric quadrupole transition rates are also discussed. We have considered isotopes for which the experimental spectrum [27] was complete enough in the sense that the first few $0^{+}, 2^{+}$, $4^{+}$, and $6^{+}$levels could be identified unambiguously. Then the $2_{1}^{+}$state was associated with the $(\xi, \tau)=(1,1)$ model state, the $4_{1}^{+}$and $2_{2}^{+}$levels were assigned to $(\xi, \tau)=(1,2)$, while the $3^{+}$and $6^{+}$members of the $(\xi, \tau)=(1,3)$ multiplet were identified with the experimental $3_{1}^{+}$and $6_{1}^{+}$states. With this it was assumed that the experimental levels are not intruder states with some other type of structure. The level assignments are displayed in Tables III, IV, and V.

Special care has to be taken in identifying the $0^{+}$levels, as these are crucial in the interpretation of quadrupole collective behavior in terms of the Bohr Hamiltonian. Both, the $E(5)$ symmetry and the sextic oscillator potential at the critical point predict that the $0^{+}$state with radially excited configuration is lower than the $0^{+}$state belonging to the multiplet with $\tau$ excitation. These states correspond to the quantum numbers $(\xi, \tau)=(2,0)$ and $(\xi, \tau)=(1,3)$, respectively, and this feature is also manifested in the electric quadrupole transitions. The first level decays dominantly into the $2_{2}^{+}$ level, while the latter one prefers decaying into the $2_{1}^{+}$state. Whenever information on the $B(E 2)$ values was available, it was used in the identification of the $0^{+}$levels in Tables III, IV,

TABLE III. Experimental levels (in $\mathrm{keV}$ ) of the $\mathrm{Ru}$ nuclei corresponding to the $\phi_{\xi, \tau}$ model states. Experimental energies in parentheses indicate ambiguous $J^{\pi}$ assignment. In case of the $0^{+}$ states, a lower index $j$ indicates their sequence, that is, $0_{j}^{+}$. Bold numbers indicate confirmed assignment of $0^{+}$levels based on $B(E 2)$ data. The next section includes the $a$ and $b$ parameters obtained from the global fit, followed by the energy levels calculated with these parameters.

\begin{tabular}{|c|c|c|c|c|c|c|}
\hline $\begin{array}{l}A \\
N\end{array}$ & $\begin{array}{c}{ }^{98} \mathrm{Ru} \\
54\end{array}$ & $\begin{array}{c}{ }^{100} \mathrm{Ru} \\
56\end{array}$ & $\begin{array}{c}{ }^{102} \mathrm{Ru} \\
58\end{array}$ & $\begin{array}{c}{ }^{104} \mathrm{Ru} \\
60\end{array}$ & $\begin{array}{c}{ }^{106} \mathrm{Ru} \\
62\end{array}$ & $\begin{array}{c}{ }_{64}^{108} \mathrm{Ru} \\
64\end{array}$ \\
\hline$E_{1,1}^{\text {Exp. }}\left(2^{+}\right)$ & 652 & 540 & 475 & 358 & 270 & 242 \\
\hline$E_{1,2}^{\text {Exp. }}\left(2^{+}\right)$ & 1414 & 1362 & 1103 & 893 & 792 & (708) \\
\hline$E_{1,2}^{\text {Exp. }}\left(4^{+}\right)$ & 1398 & 1227 & 1106 & 889 & (715) & 665 \\
\hline$E_{1,3}^{\text {Exp. }}\left(0^{+}\right)$ & $(2375)_{3}$ & $1741_{3}$ & $1837_{3}$ & $1335_{3}$ & & \\
\hline$E_{1,3}^{\text {Exp. }}\left(3^{+}\right)$ & 1797 & 1881 & 1522 & 1242 & (1092) & (975) \\
\hline$E_{1,3}^{\text {Exp. }}\left(4^{+}\right)$ & 2267 & 2063 & 1799 & 1503 & (1307) & (1183) \\
\hline$E_{1,3}^{\text {Exp. }}\left(6^{+}\right)$ & 2223 & 2076 & 1873 & 1556 & (1296) & 1241 \\
\hline$E_{2,0}^{\text {Exp. }}\left(0^{+}\right)$ & $1322_{2}$ & $\mathbf{1 1 3 0}_{2}$ & $944_{2}$ & $\mathbf{9 8 8}_{2}$ & $991_{2}$ & $(976)_{2}$ \\
\hline$a_{\text {Fit }}$ & 0 & 0 & 0 & 1279 & 4101 & 5170 \\
\hline$b_{\text {Fit }}$ & 352 & 319 & 284 & 222 & 151 & 115 \\
\hline$E_{1,1}^{\mathrm{Fit}}$ & 704 & 638 & 569 & 424 & 315 & 298 \\
\hline$E_{1,2}^{\mathrm{Fit}}$ & 1409 & 1276 & 1138 & 942 & 808 & 739 \\
\hline$E_{1,3}^{\mathrm{Fit}}$ & 2113 & 1914 & 1707 & 1385 & 1184 & 1113 \\
\hline$E_{2,0}^{\mathrm{Fit}}$ & 1409 & 1276 & 1138 & 996 & 1011 & 1019 \\
\hline
\end{tabular}

TABLE IV. The same as Table III for Pd nuclei.

\begin{tabular}{lccccc}
\hline \hline$A$ & ${ }^{102} \mathrm{Pd}$ & ${ }^{104} \mathrm{Pd}$ & ${ }^{106} \mathrm{Pd}$ & ${ }^{108} \mathrm{Pd}$ & ${ }^{110} \mathrm{Pd}$ \\
$N$ & 56 & 58 & 60 & 62 & 64 \\
\hline$E_{1,1}^{\text {Exp. }}\left(2^{+}\right)$ & 556 & 556 & 512 & 434 & 374 \\
$E_{1,2}^{\text {Exp. }}\left(2^{+}\right)$ & 1535 & 1342 & 1128 & 931 & 814 \\
$E_{1,2}^{\text {Exp. }}\left(4^{+}\right)$ & 1276 & 1324 & 1229 & 1048 & 921 \\
$E_{1,3}^{\text {Exp. }}\left(0^{+}\right)$ & $1658_{3}$ & $1793_{3}$ & $1706_{3}$ & $1314_{3}$ & $1171_{3}$ \\
$E_{1,3}^{\text {Exp. }}\left(3^{+}\right)$ & 2112 & 1821 & 1558 & 1335 & $(1212)$ \\
$E_{1,3}^{\text {Exp. }}\left(4^{+}\right)$ & 2138 & 2082 & 1932 & $(1624)$ & 1398 \\
$E_{1,3}^{\text {Exp. }}\left(6^{+}\right)$ & 2111 & 2250 & 2077 & 1771 & 1574 \\
$E_{2,0}^{\text {Exp. }}\left(0^{+}\right)$ & $1593_{2}$ & $\mathbf{1 3 3 4}$ & $\mathbf{1 1 3 4}$ & $\mathbf{1 0 5 3}$ & $\mathbf{9 4 7}$ \\
$a_{\text {Fit }}$ & 6579 & 0 & 0 & 1270 & 971 \\
$b_{\text {Fit }}$ & 303 & 331 & 299 & 239 & 216 \\
$E_{1,1}^{\text {Fit }}$ & 543 & 661 & 598 & 459 & 416 \\
$E_{1,2}^{\text {Fit }}$ & 1401 & 1322 & 1196 & 1009 & 906 \\
$E_{1,3}^{\text {Fit }}$ & 2008 & 1983 & 1795 & 1485 & 1338 \\
$E_{2,0}^{\text {Fit }}$ & 1589 & 1322 & 1196 & 1057 & 949 \\
\hline \hline
\end{tabular}

and $\mathrm{V}$. This information was also used in the level assignment in the remaining members of the isotope chains: It turned out that the energies of the $0_{2}^{+}$and $0_{3}^{+}$levels changed rather smoothly along the chains. In general, it was reasonable to keep to the concordant prediction of the $E(5)$ symmetry and the sextic oscillator and to assign the experimental $0_{2}^{+}$state to the $(\xi, \tau)=(2,0)$ model state. There is not much information on possible intruder states in this region, so we did not consider the higher-lying experimental $0^{+}$states. It is notable that in ${ }^{134} \mathrm{Ba}$, the first and perhaps the best example for $E(5)$ symmetry, the order of the $0_{2}^{+}$and $\mathrm{O}_{3}^{+}$levels, differs from the prediction of $E(5)$ symmetry.

The data set complied as above in Tables III, IV, and $\mathrm{V}$ was found to be reasonably complete: Only two $\mathrm{O}_{3}^{+}$ levels are missing and the number of states with ambiguous

TABLE V. The same as Table III for Cd nuclei.

\begin{tabular}{|c|c|c|c|c|c|c|}
\hline $\begin{array}{l}A \\
N\end{array}$ & $\begin{array}{c}{ }^{106} \mathrm{Cd} \\
58\end{array}$ & $\begin{array}{c}{ }^{108} \mathrm{Cd} \\
60\end{array}$ & $\begin{array}{c}{ }^{110} \mathrm{Cd} \\
62\end{array}$ & $\begin{array}{c}{ }^{112} \mathrm{Cd} \\
64\end{array}$ & $\begin{array}{c}{ }_{66}^{114} \mathrm{Cd} \\
66\end{array}$ & $\begin{array}{c}{ }^{116} \mathrm{Cd} \\
68\end{array}$ \\
\hline$E_{1,1}^{\text {Exp. }}\left(2^{+}\right)$ & 633 & 633 & 658 & 618 & 559 & 514 \\
\hline$E_{1,2}^{\text {Exp. }}\left(2^{+}\right)$ & 1717 & 1602 & 1476 & 1312 & 1210 & 1213 \\
\hline$E_{1,2}^{\text {Exp. }}\left(4^{+}\right)$ & 1494 & 1509 & 1543 & 1416 & 1284 & 1219 \\
\hline$E_{1,3}^{\text {Exp. }}\left(0^{+}\right)$ & $2144_{3}$ & $1913_{3}$ & $1731_{3}$ & $1433_{3}$ & $1306_{3}$ & $1283_{2}$ \\
\hline$E_{1,3}^{\text {Exp. }}\left(3^{+}\right)$ & (2254) & 2146 & 2163 & 2065 & 1864 & (1916) \\
\hline$E_{1,3}^{\text {Exp. }}\left(4^{+}\right)$ & 2105 & 2239 & 2220 & 1871 & 1732 & 2042 \\
\hline$E_{1,3}^{\text {Exp. }}\left(6^{+}\right)$ & 2503 & 2541 & 2480 & 2168 & 1990 & 2027 \\
\hline$E_{2,0}^{\text {Exp. }}\left(0^{+}\right)$ & $1795_{2}$ & $1721_{2}$ & $1473_{2}$ & $\mathbf{1 2 2 5}_{2}$ & $\mathbf{1 1 3 5}_{2}$ & $1380_{3}$ \\
\hline$a_{\text {Fit }}$ & 9252 & 7014 & 0 & 0 & 0 & 7057 \\
\hline$b_{\text {Fit }}$ & 330 & 339 & 361 & 331 & 308 & 279 \\
\hline$E_{1,1}^{\mathrm{Fit}}$ & 581 & 615 & 723 & 662 & 616 & 499 \\
\hline$E_{1,2}^{\mathrm{Fit}}$ & 1557 & 1539 & 1446 & 1324 & 1232 & 1327 \\
\hline$E_{1,3}^{\mathrm{Fit}}$ & 2217 & 2217 & 2169 & 1987 & 1848 & 1897 \\
\hline$E_{2,0}^{\mathrm{Fit}}$ & 1795 & 1721 & 1446 & 1324 & 1232 & 1540 \\
\hline
\end{tabular}


$J^{\pi}$ assignment is 13 , compared to 134 , the total number of experimental levels considered in the 17 nuclei. In the next step, the model parameters $a$ and $b$ were fitted to the experimental data for each nucleus. In this process states with ambiguous $J^{\pi}$ assignment were taken into account with weight 0.5 . A special common feature both to the $E(5)$ symmetry and to the critical point for the sextic oscillator potential in the simple formulation presented here is that the $2_{2}^{+}$and $4_{1}^{+}$levels are degenerate, as are the $3_{1}^{+}, 4_{2}^{+}, 6_{1}^{+}$, and $0_{3}^{+}\left(\right.$or $0_{2}^{+}$) levels. This degeneracy could be removed, including, for instance, a $J^{2}$ term in the Hamiltonian, but this implies an extra parameter, and we prefer to keep the formalism as simple as possible. Obviously, this degeneracy is not fulfilled in the experimental spectrum, so the fit concerned an average value of these energy levels. Although because of the experimental ambiguities we did not make efforts to fit the $(\xi, \tau)=(2,1) 2^{+}$states, their energy can be calculated using Table I after $a$ and $b$ have been determined. We shall do this only in the case of a few selected illustrative examples, where $E 2$ transitions from this state to the $(\xi, \tau)=(2,0) 0^{+}$state are known.

As has been discussed before, a first approximation of the parameters can be given by the exact formulas in Eq. (8). The only ambiguity here is the choice of the $(\xi, \tau)=(1,2)$ level, that is, whether the $2_{2}^{+}$or the $4_{1}^{+}$level is considered. The preceding formulas also indicate that whenever the $(\xi, \tau)=(2,0)$ (generally the experimental $\left.0_{2}^{+}\right)$state is below the $(\xi, \tau)=(1,2)$ (i.e., the $2_{2}^{+}$or $4_{1}^{+}$level), then the fitted $a$ parameter turns out to be negative, which is incompatible with the normalizability of the wave functions [Eq. (6)]. This situation also occurred in the general fits. Because the $a$ parameter obtained from the fit was usually a small negative number, in these cases we applied the harmonic approximation $a=0$ and fitted only the $b$ parameter. The fitted parameters are displayed in the second part of Tables III, IV, and V, followed by the fitted energy eigenvalues.

\section{DISCUSSION}

As expected from the relatively smooth change of the individual energy levels along the isotope chains, the fitted parameters also exhibit a relatively smooth behavior from nucleus to nucleus. This is especially so for the Ru chain: $a$ and $b$ move monotonously with increasing $A$. Furthermore, as can be seen in Fig. 3, in the process the chain crosses the parabola separating the shape phases with spherical and deformed potential minimum. In fact, it is the ${ }^{104} \mathrm{Ru}$ nucleus that is the closest to the parabola, which is in agreement with the findings of Ref. [10]. The light isotopes not only have spherical potential minimum, but are also close to the harmonic limit. This is also indicated by the finding that the $2_{2}^{+}, 4_{1}^{+}$, and $0_{2}^{+}$levels are close in energy, which is roughly double the excitation energy of the $2_{1}^{+}$level. The evolution of the potential shape along the $\mathrm{Ru}$ chain is shown in Fig. 4. The potential curve for the lighter isotopes is parabolic; that is, it corresponds to $a=0$ for reasons outlined in Sec. IV. At ${ }^{104} \mathrm{Ru}$ the potential curve gets flatter near the origin, which is still the potential minimum. Then at ${ }^{106} \mathrm{Ru}$ a deformed minimum appears, which moves further away from the origin at ${ }^{108} \mathrm{Ru} .{ }^{104} \mathrm{Ru}$ is selected as the critical

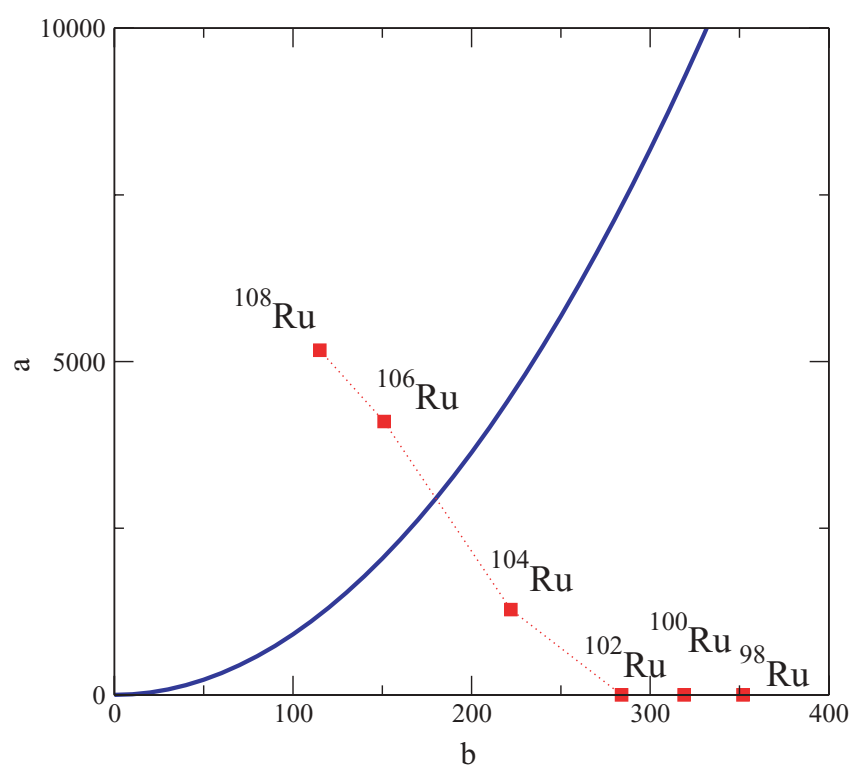

FIG. 3. (Color online) Evolution of the potential parameters along the $\mathrm{Ru}$ chain in the model space.

nucleus in this isotope chain with the criterion, usually used in the literature, of being the closest isotope (flattest potential) to the critical point (critical parabola in the sextic potential case) keeping spherical shape. The spectroscopic properties of the ${ }^{104} \mathrm{Ru}$ nucleus are summarized in Fig. 5. It is seen that the energy eigenvalues are reproduced reasonably well, while the $B(E 2)$ values come out somewhat weaker compared to the strength of the transition from the $2_{1}^{+}$state. It has to be noted though that the $B(E 2)$ values are genuine predictions in the sense that they involve no further parameter in addition to $a$ and $b$ determined from the fit to the energy spectrum. The $B(E 2)$ values predicted by the $E(5)$ symmetry [10] are usually somewhat weaker than the present values.

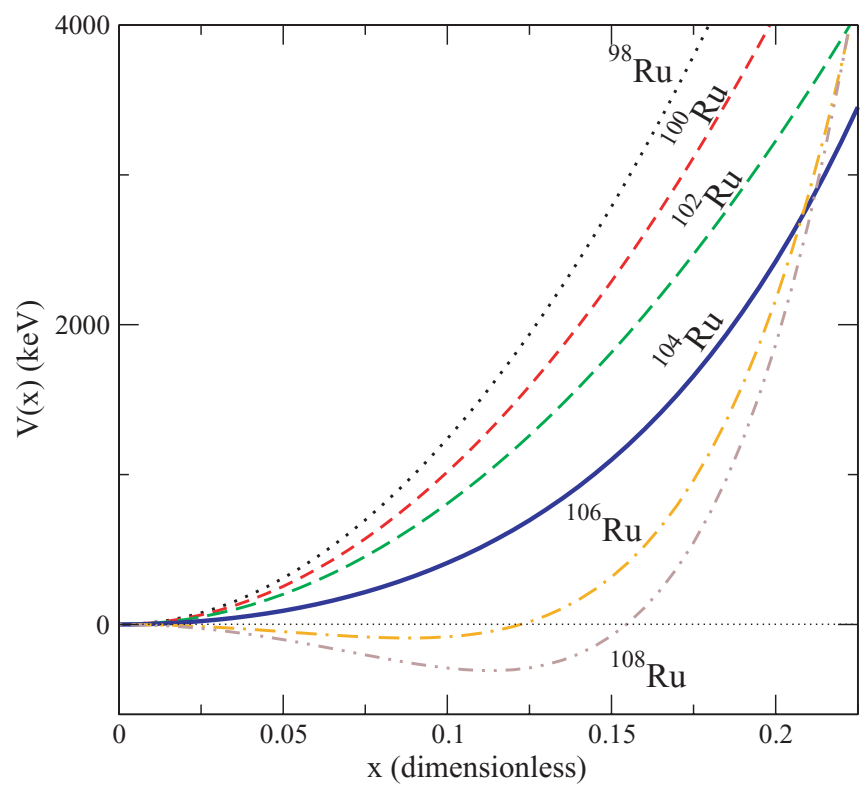

FIG. 4. (Color online) Evolution of the potential energy surface along the $\mathrm{Ru}$ isotope chain. 


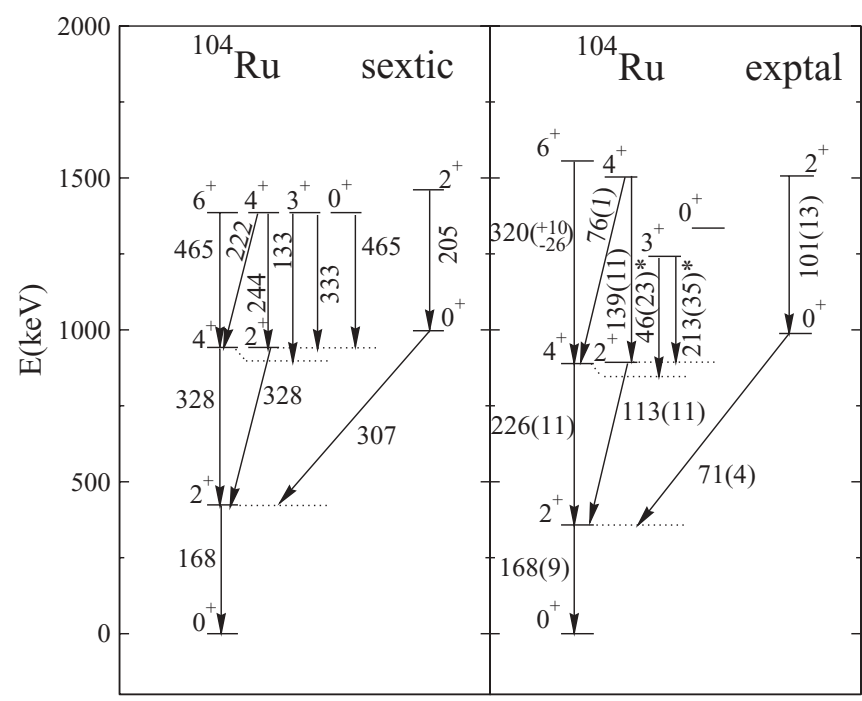

FIG. 5. Spectroscopic properties of the ${ }^{104} \mathrm{Ru}$ nucleus and their interpretation in terms of the sextic oscillator model. The experimental data are taken from Ref. [28], except those marked with an asterisk, which are taken from Ref. [29]. $B(E 2)$ units are $10^{-3} \mathrm{e}^{2}$ $\mathrm{b}^{2}$. The theoretical transition probability $B\left(E 2 ; 2_{1}^{+} \rightarrow 0_{1}^{+}\right)$has been normalized to the experimental value.

The situation is somewhat different in the case of the $\mathrm{Pd}$ isotopes. As it can be seen from Fig. 6, in this case the points indicating the isotopes all remain in the domain to the right of the critical parabola. This means that the spectra of all nuclei yield parameters corresponding to a spherical minimum, although the ${ }^{102} \mathrm{Pd}$ nucleus lies rather close to the critical parabola separating the spherical and deformed shape phases. The situation is further complicated by the fact that the energy spectrum of the ${ }^{104} \mathrm{Pd}$ and ${ }^{106} \mathrm{Pd}$ nuclei have to be approximated by a harmonic oscillator configuration, because the $0_{2}^{+}$state lies slightly below the $4_{1}^{+}$level, leading formally

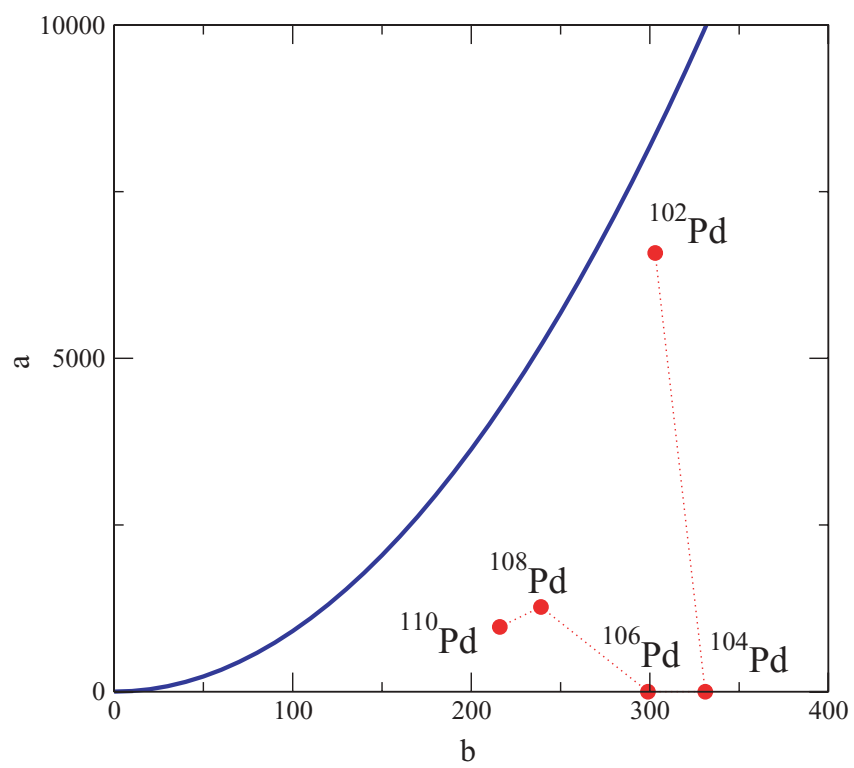

FIG. 6. (Color online) Evolution of the potential parameters along the Pd chain in the model space.

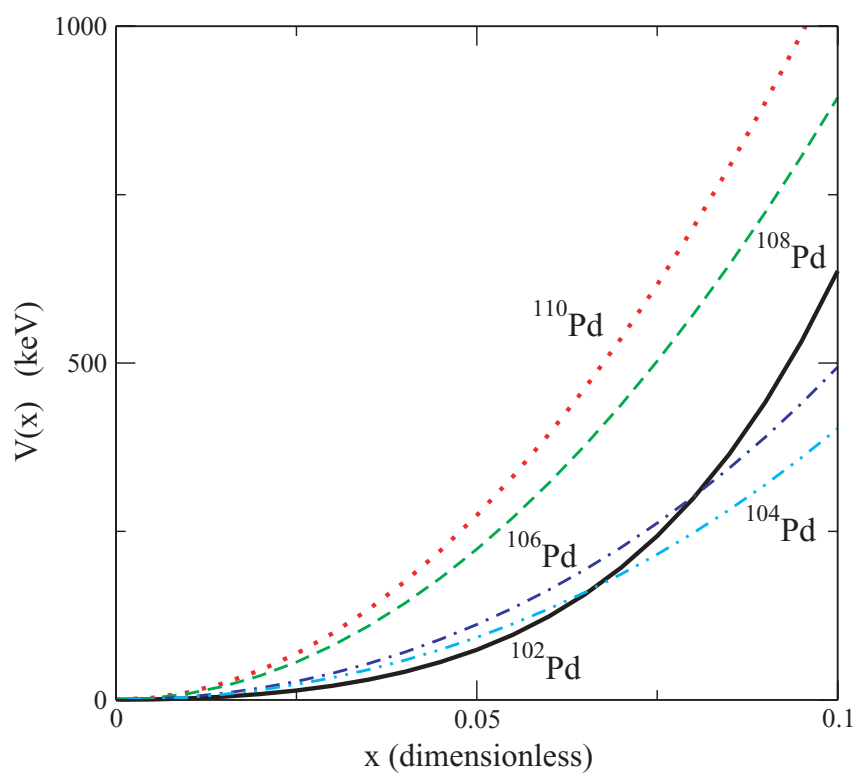

FIG. 7. (Color online) Evolution of the potential surface along the $\mathrm{Pd}$ isotope chain.

to an unacceptable small negative value of $a$. In accordance with previous findings [11], it is the ${ }^{102} \mathrm{Pd}$ nucleus that is the closest to the critical parabola [which is expected to be close to the $E(5)$ situation]. In Fig. 7 the evolution of the potential surface for the Pd isotopes is presented. It is seen that ${ }^{102} \mathrm{Pd}$ is the isotope with flatter potential close to the origin, as expected for a critical nucleus in a second-order shape phase transition. In Fig. 8 the experimental and calculated spectra for ${ }^{102} \mathrm{Pd}$ is presented including the $E 2$ probabilities. Again, the agreement of the calculation for energies and $B(E 2)$ 's is reasonable: Though some transitions are weaker than the experimental values and the $E(5)$ predictions [12], in some cases [e.g.,

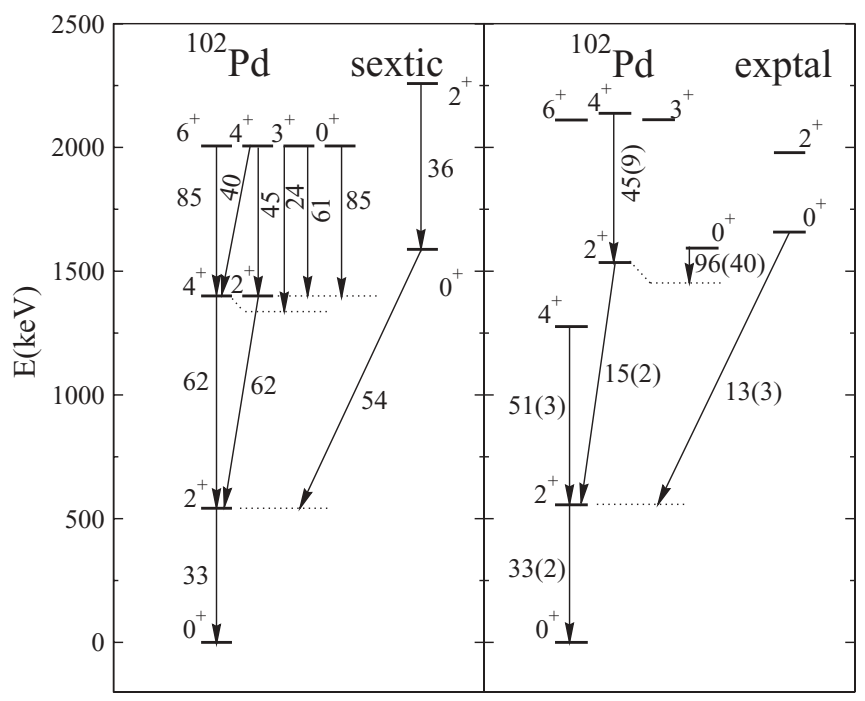

FIG. 8. Spectroscopic properties of the ${ }^{102} \mathrm{Pd}$ nucleus and their interpretation in terms of the sextic oscillator model. Theoretical $E 2$ transition probabilities are normalized to the experimental value for the $B\left(E 2 ; 2_{1}^{+} \rightarrow 0_{1}^{+}\right)=33$ W.u. Data are taken from Refs. [11,30,31]. 


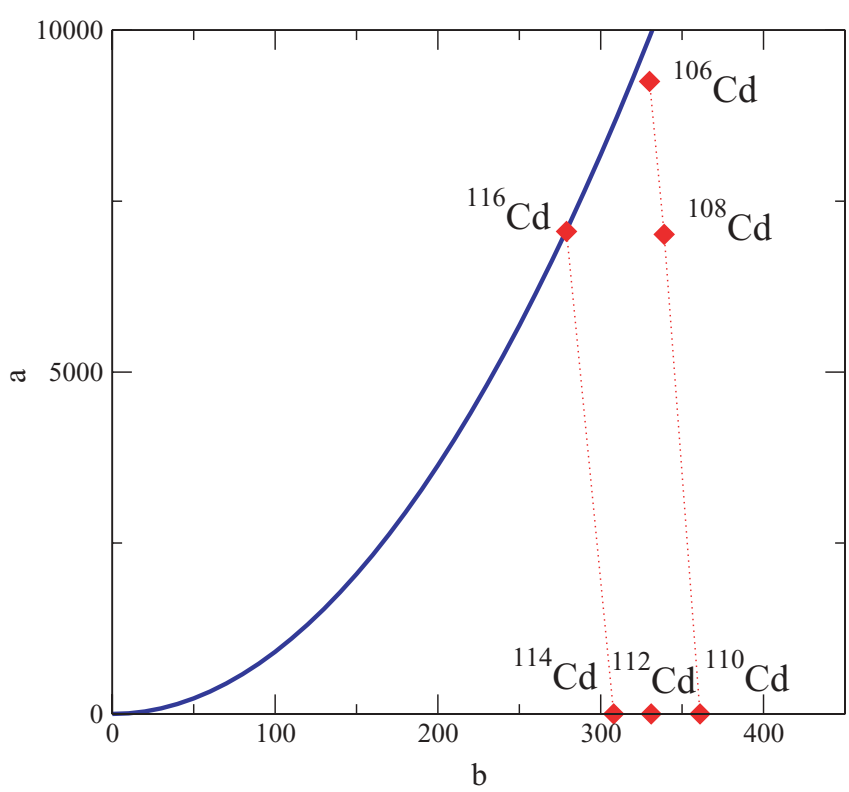

FIG. 9. (Color online) Evolution of the potential parameters along the $\mathrm{Cd}$ isotope chain.

transitions from the $(\xi, \tau)=(1,3)$ states] the agreement is good.

The situation is rather similar in the case of the Cd isotope chain, as can be seen from Fig. 9: For the light isotopes, the trajectory starts near the critical parabola $\left({ }^{106,108} \mathrm{Cd}\right)$, then proceeds along the $b$ axis corresponding to the harmonic approximation $\left({ }^{110-114} \mathrm{Cd}\right.$ for reasons similar to those explained in the case of the $\mathrm{Pd}$ nuclei), and finally returns close to the critical parabola $\left({ }^{116} \mathrm{Cd}\right)$. In fact, the ${ }^{116} \mathrm{Cd}$ nucleus is the closest to the critical parabola; however, this nucleus differs from the remaining $\mathrm{Cd}$ isotopes in that the $(\xi, \tau)=(2,0)$ model state is assigned to the $0_{3}^{+}$experimental level, rather than to $0_{2}^{+}$. Nevertheless, this choice is justified by the relative magnitude of the $B(E 2)$ rates between $0^{+}$and $2^{+}$states. The evolution of the potential energy function for the $\mathrm{Cd}$ isotopes is presented in Fig. 10. Again, the flatter surface close to the origin corresponds to ${ }^{116} \mathrm{Cd}$ as expected for a critical nucleus in the transition under study. It should be noted that the isotopes ${ }^{106,108} \mathrm{Cd}$ which have been proposed as examples of $E(5)$ symmetry [12] are very close to the parabola, as can be seen in Fig. 9 and, consequently, have energy surfaces that are quite flat close to the origin, as shown in Fig. 10. This justifies that they have been proposed as E(5)-type nuclei. In Fig. 11 the spectroscopic properties of the ${ }^{116} \mathrm{Cd}$ nucleus and their interpretation in terms of the sextic oscillator model are displayed in Fig. 11. Again, the agreement of the calculation for excitation energies and $B(E 2)$ 's is reasonable. In particular, the sequence of transitions from the $6_{1}^{+}$state to the ground state is reproduced rather well, as are transitions from the $3_{1}^{+}$ state. The transition from the $2_{2}^{+}$to the $2_{1}^{+}$state is predicted to be stronger than the experimental value, which is similar to the situation observed for other critical-point nuclei $\left({ }^{102} \mathrm{Pd}\right.$, ${ }^{106} \mathrm{Cd},{ }^{108} \mathrm{Cd}[12]$, and $\left.{ }^{104} \mathrm{Ru}[10]\right)$ in this region. The transition from the radially excited $0^{+}$state to $2_{1}^{+}$is also predicted to be stronger than the experimental value; however, the difference

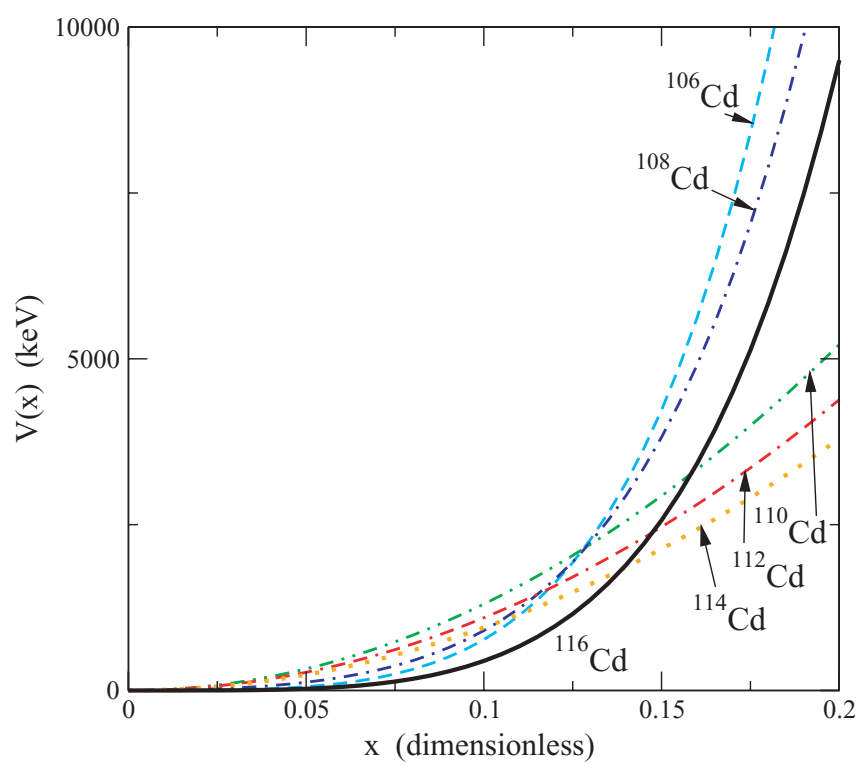

FIG. 10. (Color online) Evolution of the potential energy surface along the $\mathrm{Cd}$ isotope chain.

is smaller than in the case of ${ }^{102} \mathrm{Pd}$ and ${ }^{104} \mathrm{Ru}$. (For the ${ }^{106} \mathrm{Cd}$ and ${ }^{108} \mathrm{Cd}$ isotopes the precise $B(E 2)$ values for this transition are unknown.)

It is remarkable that the best candidates proposed previously [10-12] for nuclei close to the phase transition from the spherical to the deformed domain turned out to be good candidates for this within the framework of the sextic oscillator model, too.

It has to be noted that we did not fit the $B$ inertia parameter in Eq. (1), although it is known to vary with the mass $A$ approximately as $B \sim A^{5 / 3}$ [1]. Neglecting this effect does not influence the results significantly within the domain

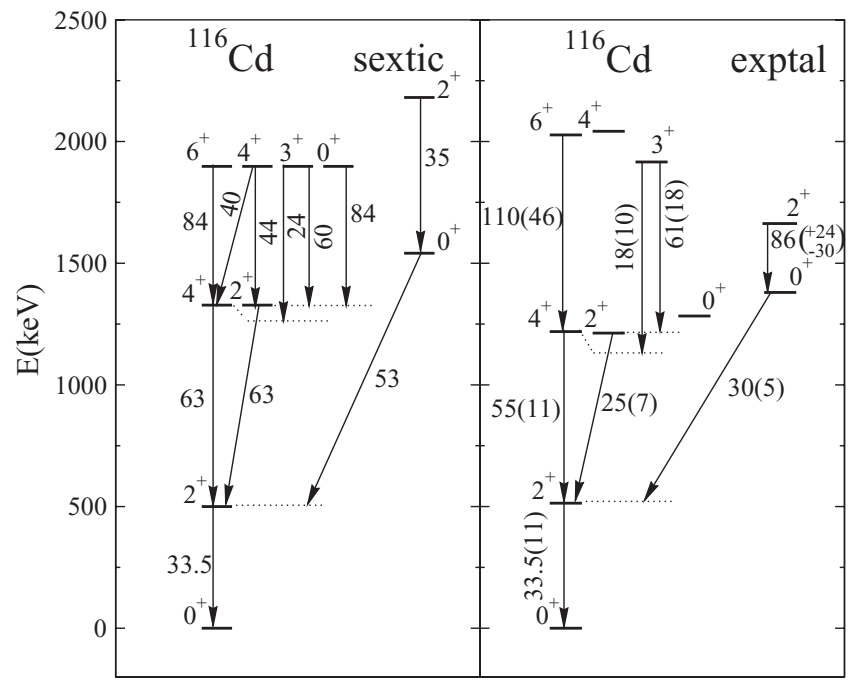

FIG. 11. Spectroscopic properties of the ${ }^{116} \mathrm{Cd}$ nucleus and their interpretation in terms of the sextic oscillator model. Theoretical $E 2$ transitions are normalized to the experimental value for the $B\left(E 2 ; 2_{1}^{+} \rightarrow 0_{1}^{+}\right)=33.5$ W.u. Data are taken from Ref. [32] and references therein. 
TABLE VI. The ratio $\rho^{2}\left(E 0 ; 2_{2,1}^{+} \rightarrow 2_{1,1}^{+}\right) / \rho^{2}\left(E 0 ; 0_{2,0}^{+} \rightarrow 0_{1,0}^{+}\right)$ of selected $E 0$ transition strengths. For the sake of compact presentation, data are arranged into columns corresponding to $N-Z$.

\begin{tabular}{lccccccc}
\hline \hline & $Z / N-Z$ & 10 & 12 & 14 & 16 & 18 & 20 \\
\hline $\mathrm{Ru}$ & 44 & 1.4 & 1.4 & 1.4 & 1.3146 & 1.1516 & 1.0995 \\
$\mathrm{Pd}$ & 46 & 1.2324 & 1.4 & 1.4 & 1.3244 & 1.3283 & - \\
$\mathrm{Cd}$ & 48 & 1.2168 & 1.2468 & 1.4 & 1.4 & 1.4 & 1.2109 \\
\hline \hline
\end{tabular}

considered here. The handling of this degree of freedom is possible by a rescaling of $a, b$, and $c^{ \pm}$. However, rescaling $c^{ \pm}$means that the critical parabola will be different for each nucleus.

Although there are few experimental data on the $B(E 0)$ values, we present the relative strength of two such transitions in each nucleus considered here. Comparison of these values with those obtained from other models may be instructive. The magnitude usually measured experimentally is the $E 0$ transition strength $\rho^{2}(E 0)$ defined as

$\rho_{i, f}^{2}(\mathrm{E} 0)=\left|\frac{\left\langle\text { final }\left|T^{(\mathrm{E} 0)}\right| \text { initial }\right\rangle}{e R^{2}}\right|^{2}, \quad$ with $\quad R \approx 1.2 A^{1 / 3} \mathrm{fm}$.

With the formalism presented here, ratios of $\rho^{2}(\mathrm{E} 0)$ can be easily calculated. In Table VI the ratio $\rho^{2}\left(E 0 ; 2_{2,1}^{+} \rightarrow\right.$ $\left.2_{1,1}^{+}\right) / \rho^{2}\left(E 0 ; 0_{2,0}^{+} \rightarrow 0_{1,0}^{+}\right)$is presented for each isotope chain. The value of this ratio at the critical parabola is 1.2107. The only available experimental data for this ratio are [32] 0.84(60) for ${ }^{112} \mathrm{Cd}$ and $2.25(34)$ for ${ }^{114} \mathrm{Cd}$.

\section{SUMMARY}

In this work we have exploited the possibility of describing critical nuclei in the transition from spherical to $\gamma$-unstable shapes by using a sextic oscillator potential in the corresponding Bohr equation. For this potential, exact analytical results can be obtained for a relevant selected number of lowlying collective states. These results include energies, wave functions, and electric quadrupole and monopole transitions. Close analytical expressions for all of them are provided in this article. Within this formalism, all magnitudes depend basically on two parameters $(a, b)$. The critical point of interest is located in a parabola $b^{2}=11 a$. All points in this parabola give the same ratios for energies and $B(E 2)$ 's, thus providing benchmark numbers for energy and $B(E 2)$ ratios at the critical points. These numbers can be used to identify critical nuclei within the chart of nuclei. In this article the isotope chains of $\mathrm{Ru}, \mathrm{Pd}$, and $\mathrm{Cd}$ isotopes have been studied as an example. Our study confirms that ${ }^{104} \mathrm{Ru},{ }^{102} \mathrm{Pd}$, and ${ }^{106,108} \mathrm{Cd}$ are good candidates as critical nuclei. In addition, ${ }^{116} \mathrm{Cd}$ is proposed as a new critical nucleus. It would be worthwhile to study this isotope within the framework of $E(5)$ symmetry too.

A possible task for the future is developing the formalism to treat nuclei with X(5) symmetry. For this, the $\gamma$ degrees of freedom also have to be incorporated into the potential. There are several ways of approximating this effect $[14,33,34]$ so that the application of the sextic oscillator to $\mathrm{X}(5)$ nuclei seems not only desirable but also possible. For this, potential shapes with two minima (corresponding to $b<0$ and $b^{2}>4 a c^{\pi}$ ) seem suitable.

\section{ACKNOWLEDGMENTS}

This work was supported by OTKA Grants No. T49646 and K72357 (Hungary), the Spanish MEC, and the European regional development fund (FEDER) under projects FIS2008-04189 and CPAN-Ingenio, and by the Junta de Andalucía. G. L. is grateful for hospitality at the University of Sevilla.

\section{APPENDIX: MATRIX ELEMENTS OF THE SEXTIC WAVE FUNCTIONS}

The integrals necessary to evaluate the matrix elements can be calculated in terms of parabolic cylinder functions using Eq. 2.3.15.3 of Ref. [35], and the resulting formula can be rewritten in terms of confluent hypergeometric functions applying Eqs. 19.3.1 and 19.12.4 of Ref. [22]. The final expression is then

$$
\begin{aligned}
I^{(A)} & =\int_{0}^{\infty} \beta^{A} \exp \left(-\frac{a}{2} \beta^{4}-b \beta^{2}\right) d \beta \\
& =\frac{1}{2} \Gamma\left(\frac{A+1}{2}\right)(2 a)^{-(A+1) / 4} U\left(\frac{A+1}{4}, \frac{1}{2} ; \frac{b^{2}}{2 a}\right) .
\end{aligned}
$$

This equation is valid for $a>0$. For $a=0$, the problem reduces to the harmonic oscillator, so everything can be taken from the standard treatment of that problem. Note that there was a minor typing error in Eq. (11) of Ref. [16] in the power of $2 a$.

The $U(s, t ; z)$ Kummer's functions can be expressed in terms of the usual confluent hypergeometric functions as

$$
\begin{aligned}
U\left(s, \frac{1}{2}, z\right)= & \frac{\pi^{1 / 2}}{\Gamma\left(s+\frac{1}{2}\right)}{ }_{1} F_{1}\left(s, \frac{1}{2} ; z\right) \\
& -\frac{2 \pi^{1 / 2}}{\Gamma(s)}{ }_{1} F_{1}\left(s+\frac{1}{2}, \frac{3}{2} ; z\right)
\end{aligned}
$$

(see Eq. 13.1.3 of Ref. [22]). We also display two more equations from Ref. [22], which play a role in our calculations:

$$
U(s, t ; z)=z^{1-t} U(1+s-t, 2-t ; z)
$$

(Eq. 13.1.29) and

$$
(t-s) U(s, t ; z)+U(s-1, t ; z)-z U(s, t+1 ; z)=0
$$

(Eq. 13.4.18). 
Taking the general matrix elements with the wave functions [Eq. (6)] and applying Eq. (A2), we get the general expression

$$
\begin{aligned}
W_{\alpha, \gamma}^{\delta} \equiv & \int_{0}^{\infty} \phi_{\gamma}(\beta) \beta^{\delta} \phi_{\alpha}(\beta) \mathrm{d} \beta \\
= & N_{\alpha} N_{\gamma} \frac{1}{2} \Gamma\left(\frac{p_{\alpha}+p_{\gamma}+\delta+1}{2}\right)(2 a)^{-\left(p_{\alpha}+p_{\gamma}+\delta+1\right) / 4} \\
& \times\left\{U\left(\frac{1}{4}\left(p_{\alpha}+p_{\gamma}+\delta+1\right), \frac{1}{2} ; \frac{b^{2}}{2 a}\right)\right.
\end{aligned}
$$

[1] A. Bohr and B. Mottelson, Nuclear Structure (Benjamin, Reading, MA, 1975), Vol. II.

[2] F. Iachello and A. Arima, The Interacting Boson Mode (Cambridge University Press, Cambridge, UK, 1987).

[3] R. F. Casten, Nat. Phys. 2, 811 (2006).

[4] R. F. Casten and E. A. McCutchan, J. Phys. G 34, R285 (2007).

[5] D. Bonatsos, D. Lenis, and D. Petrellis, Rom. Rep. Phys. 59, 273 (2007).

[6] R. F. Casten, Prog. Part. Nucl. Phys. 62, 183 (2009).

[7] P. Cejnar and J. Jolie, Prog. Part. Nucl. Phys. 62, 210 (2009).

[8] F. Iachello, Phys. Rev. Lett. 85, 3580 (2000).

[9] R. F. Casten and N. V. Zamfir, Phys. Rev. Lett. 85, 3584 (2000).

[10] A. Frank, C. E. Alonso, and J. M. Arias, Phys. Rev. C 65, 014301 (2001).

[11] N. V. Zamfir, Phys. Rev. C 65, 044325 (2002).

[12] R. M. Clark, M. Cromaz, M. A. Deleplanque, M. Descovich, R. M. Diamond, P. Fallon, I. Y. Lee, A. O. Macchiavelli, H. Mahmud, E. Rodriguez-Vieitez, F. S. Stephens, and D. Ward, Phys. Rev. C 69, 064322 (2004).

[13] N. Pietralla (private communication).

[14] F. Iachello, Phys. Rev. Lett. 87, 052502 (2001).

[15] L. Fortunato, Eur. Phys. J. A 26, 1 (2005).

[16] G. Lévai and J. M. Arias, Phys. Rev. C 69, 014304 (2004).

[17] G. Lévai, Rev. Mex. Fis. S 52, 75 (2006).

[18] G. Lévai, AIP Conf. Proc. 1165, 211 (2009).

$$
\begin{aligned}
& +\frac{p_{\alpha}+p_{\gamma}+\delta+1}{2} \frac{d_{\alpha}+d_{\gamma}}{(2 a)^{1 / 2}} \\
& \times U\left(\frac{1}{4}\left(p_{\alpha}+p_{\gamma}+\delta+3\right), \frac{1}{2} ; \frac{b^{2}}{2 a}\right) \\
& +\frac{p_{\alpha}+p_{\gamma}+\delta+1}{2} \frac{p_{\alpha}+p_{\gamma}+\delta+3}{2} \frac{d_{\alpha} d_{\gamma}}{2 a} \\
& \left.\times U\left(\frac{1}{4}\left(p_{\alpha}+p_{\gamma}+\delta+5\right), \frac{1}{2} ; \frac{b^{2}}{2 a}\right)\right\},
\end{aligned}
$$

where $\alpha$ and $\gamma$ are a shorthand notation for $\xi, \tau$.

[19] D. R. Bès, Nucl. Phys. A 10, 373 (1959).

[20] M. A. Caprio, D. J. Rowe, and T. A. Welsh, Comput. Phys. Commun. 180, 1150 (2009).

[21] A. G. Ushveridze, Quasi-exactly Solvable Models in Quantum Mechanics (IOP Publishing, Bristol, 1994).

[22] M. Abramowitz and I. A. Stegun, Handbook of Mathematical Functions (Dover, New York, 1970).

[23] L. Wilets and M. Jean, Phys. Rev. 102, 788 (1956).

[24] J. M. Arias, Phys. Rev. C 63, 034308 (2001).

[25] D. Bonatsos, D. Lenis, N. Minkov, P. P. Raychev, and P. A. Terziev, Phys. Rev. C 69, 044316 (2004).

[26] G. Lévai, J. Phys. Conf. Ser. 205, 012023 (2010).

[27] ISOTOPE EXPLORER 3.0 $\beta 1$, http://ie.lbl.gov/ensdf/.

[28] J. Srebrny et al., Nucl. Phys. A 766, 25 (2006).

[29] J. Stachel et al., Nucl. Phys. A 419, 589 (1984).

[30] M. Luontama et al., Z. Phys. A 324, 317 (1986).

[31] D. de Frenne and E. Jacobs, Nucl. Data Sheets 83, 535 (1998).

[32] P. E. Garrett, K. L. Green, and J. L. Wood, Phys. Rev. C 78, 044307 (2008)

[33] S. De Baerdemacker, L. Fortunato, V. Hellemans, and K. Heyde, Nucl. Phys. A 769, 16 (2006).

[34] L. Fortunato, S. De Baerdemacker, and K. Heyde, Phys. Rev. C 74, 014310 (2006).

[35] A. P. Prudnikov, Yu. A. Brychkov, and O. I. Marichev, Integrals and Series (Gordon and Breach, New York, 1986), Vol. 1. 